\title{
Huge Cutaneous Metastases From Gastric Adenocarcinoma: Report of
}

\section{a Rare Case}

\author{
Ahmad R Mafi (iD) 1, * \\ ${ }^{1}$ Imam Hossein Hospital, Shahid Beheshti University of Medical Sciences, Tehran, Iran \\ "Corresponding author: Imam Hossein Hospital, Shahid Beheshti University of Medical Sciences, Tehran, Iran. Email: ahmadrmafi@yahoo.com \\ Received 2020 March 01; Revised 2020 April 08; Accepted 2020 April 20.
}

\begin{abstract}
Cutaneous metastases from gastric cancer are quite rare, accounting for only $6 \%$ of all skin metastases in males and $1 \%$ in females. In case of occurrence, they are usually a sign of advanced disease and poor prognosis. This report presents a rare case of a 27-year-old male with gastric cancer who developed huge skin metastases and surprisingly, had a few visceral metastases. After four cycles of palliative chemotherapy, he discontinued the treatment and started to consume some herbal medicine. As he had a few visceral metastases, we presume that he passed away due to septic complications of the skin lesions rather than visceral crisis.
\end{abstract}

Keywords: Gastric Cancer, Skin, Cutaneous, Metastasis

\section{Introduction}

Cutaneous metastasis from internal malignancies is relatively uncommon. It is estimated that about $0.7 \%$ $10.4 \%$ of patients with different malignancies might develop skin metastasis at some point during their illness (1). Cutaneous involvement from gastric carcinoma is quite a rare finding as only $6 \%$ of all skin metastases in males and $1 \%$ in females have been reported to arise from gastric cancer (2). It usually affects the areas close to the primary tumor (abdominal wall) and often manifests as non specific nodules (3).

Here, we report a rare case of cutaneous metastases from gastric adenocarcinoma, which was clinically presented by multiple huge erythematous skin nodules and tumors affecting both anterior and posterior neck and chest wall, and scalp.

\section{Case Presentation}

A 27-year-old man presented to our clinic with the diagnosis of gastric adenocarcinoma. Staging work up showed two metastatic lesions in the liver that were confirmed by biopsy. He and his father were illiterate and came from a remote village in the center of Iran. Although it was difficult to take an accurate family history, it seemed that he was the first member of the family with such a problem. No genetic counselling was done as they refused to travel to Tehran.
Palliative chemotherapy was started. After 4th cycle of chemotherapy, he did not continue his treatment and returned after 11 months.

At that time, he appeared ill and had lost more than 20 $\mathrm{kg}$ of his weight, and was complaining of skin lesions that had grown over the last 4 months on his chest, neck and head. On physical examination, huge erythematous skin nodules and tumors could be seen on both anterior and posterior sides of his chest and neck as well as a large tumor on his scalp (Figures 1-3).

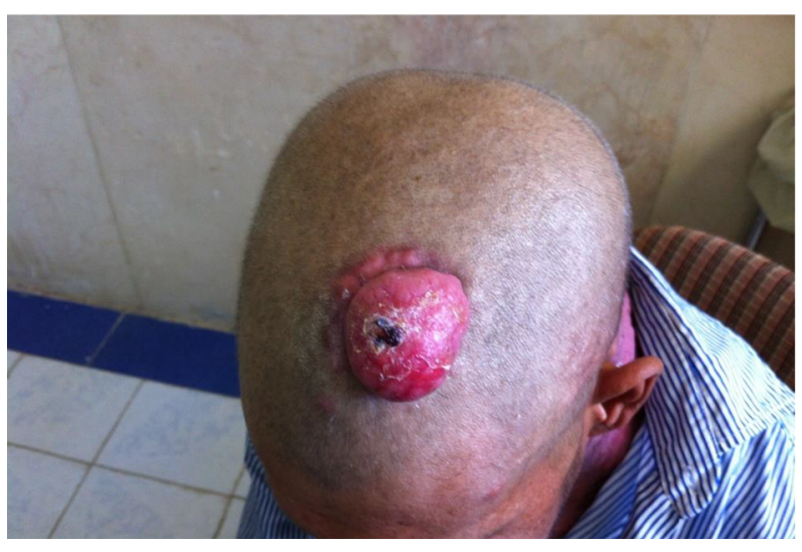

Figure 1. Skin metastasis in scalp

He mentioned that the problem had started as small 
erythematous lesions on his chest and had progressed over a few months. Several biopsies were taken, and the pathology report confirmed the diagnosis of metastatic signet ring cell (adeno) carcinoma of gastric origin. Sur-

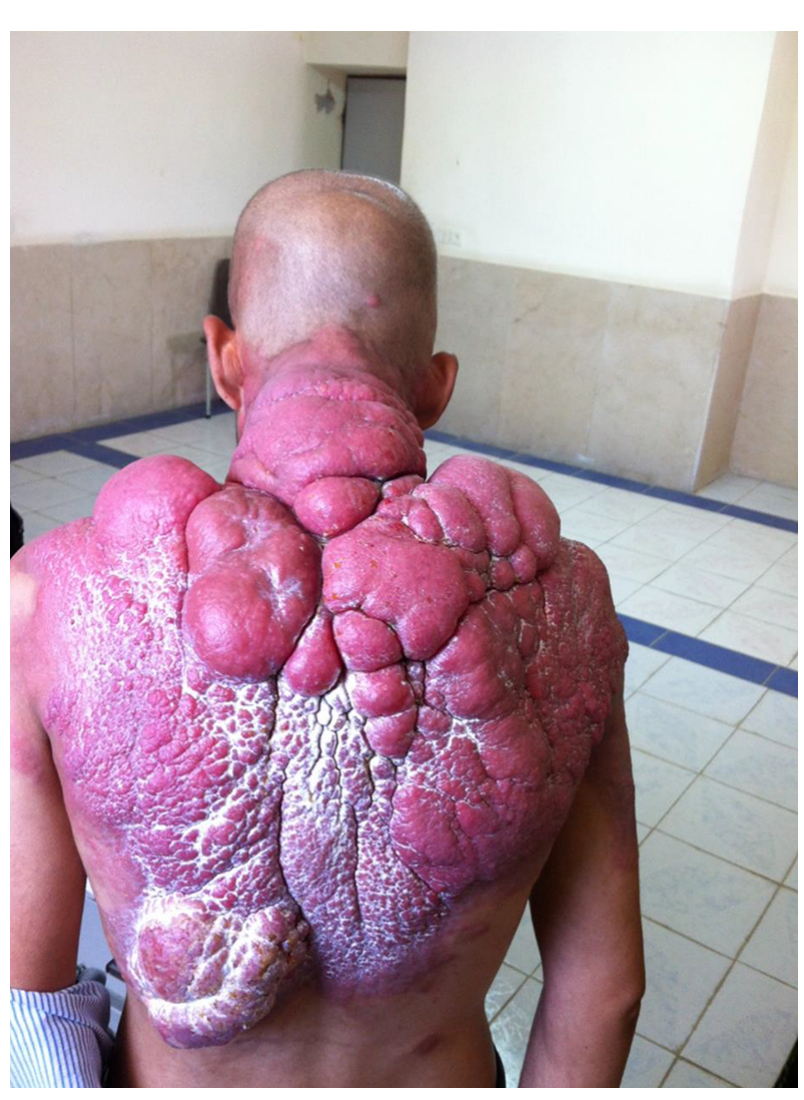

Figure 2. Huge tumor in posterior neck and chest

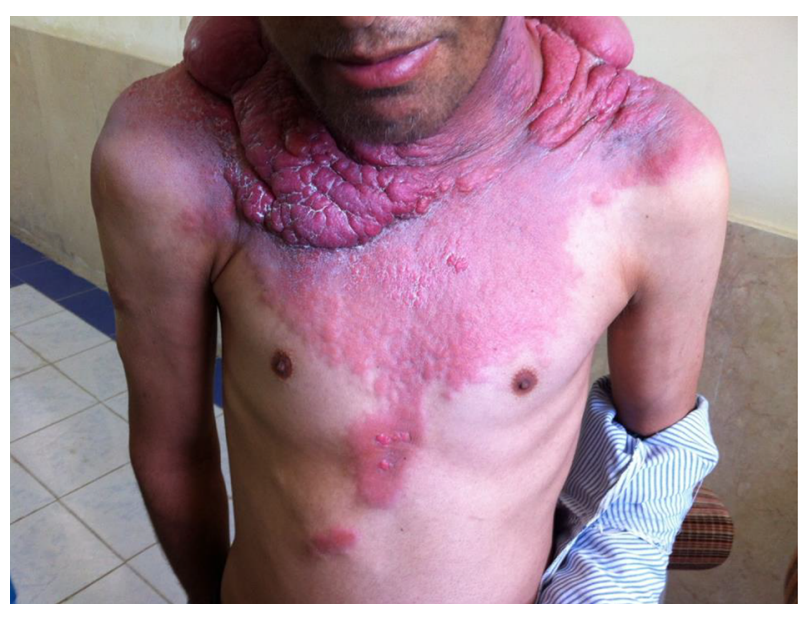

Figure 3. Huge tumor in anterior neck and chest prisingly, computed tomography of chest, abdomen and pelvis showed stable disease, with no visceral metastatic progression. Palliative chemotherapy was recommended; nevertheless, he refused the treatment and preferred to continue using the herbal medicine that he had been using since the lesions had developed. He did not come back for his visits, and by telephone follow-up we were informed by his father that he had passed away a couple of months after his last visit.

\section{Discussion}

Skin is an uncommon site for distant metastasis, and presence of skin metastasis is usually a sign of advanced disease with a poor prognosis (4). Among different malignancies, cancers of breast, lung, oral mucosa and colorectal cancer are most likely to metastasize to the skin (3). A well-known example of skin involvement from gastrointestinal cancers is Sister Mary Joseph's nodule (SMJN), which usually manifests as a cutaneous nodule on the umbilicus. SMJN accounts for about $60 \%$ of all malignant umbilical tumors (5). Metastatic involvement of the skin usually develops by direct invasion of the nearby tumor such as skin involvement in breast cancer, which often occurs in the overlying skin of the involved breast (1).

Skin metastasis can also occur through the lymphatic or hematogenous spread, and according to the literature, most of the cases of skin metastases with gastric origin have been observed in skin tissue of the abdominal wall (1, 3).

Skin metastases in our case had unusual sites of involvement as well as extraordinary huge sizes. Interestingly, although in the majority of cases skin involvement is a sign of advanced disease, our patient's visceral disease remained stable despite significant progression of the skin metastases. We were informed by his father that he passed away a couple of months after the last visit with progressive ulceration and infection of skin lesions. As he had only two hepatic metastatic lesions that had been stable for about a year, we assumed that the reason of death probably was septic complications of the skin lesions rather than visceral crisis. This patient developed gastric cancer in a very young age with unusual presentations. He and his family needed a thorough history taking and genetic counselling, however; cultural barriers, negligence and wrong beliefs about the efficacy and indications of herbal medicines, resulted in suboptimal management for his disease.

\section{Footnotes}

Authors' Contribution: Ahmad R Mafi developed the original idea and wrote the whole manuscript 
Conflict of Interests: There are no conflicts of interest. Funding/Support: None.

Informed Consent: The pictures have been taken by the patient's (and his father) verbal permission, and his face is not shown in the pictures. Unfortunately he has passed away and it is not possible to take any written consent from his father now as they live in a remote village in Kohgilooyeh Province.

\section{References}

1. Abbasi A, Abbasi F, Mahmodlou R, Mehdipour E. Cutaneous metastasis of gastric carcinoma: A rare case with unusual presentation site. Indian J Dermatopathol Diagn Dermatol. 2018;5(1). doi: 10.4103/ijdpdd.ijdpdd_38_17.
2. Xavier MH, Vergueiro Tde R, Vilar EG, Pinto JM, Issa MC, Pereira GB, et al. Cutaneous metastasis of gastric adenocarcinoma: An exuberant and unusual clinical presentation. Dermatol Online J.2008;14(11):8. [PubMed: 19094846].

3. Du C, Hong R, Liu Y, Wang J, Zhang H, Yu X. Scalp metastasis from gastric cancer: A case report and literature review. Oncol Lett. 2015;9(2):641-4. doi: 10.3892/ol.2014.2708. [PubMed: 25624893]. [PubMed Central: PMC4301561].

4. Cesaretti M, Malerba M, Basso V, Boccardo C, Santoni R, D’Alessandro $\mathrm{G}$, et al. Cutaneous metastasis from primary gastric cancer: A case report and review of the literature. Cutis. 2014;93(4):E9-E13. [PubMed: 24818191].

5. Kirchberger MC. Unusual presentation of a cutaneous metastasis in the face arising from gastric cancer: A case report. SAGE Open Med Case Rep. 2018;6:2050313X18795080. doi: 10.1177/2050313X18795080. [PubMed: 30214808]. [PubMed Central: PMC6134480]. 